\title{
La propiedad intelectual en las universidades brasileñas: Universidad Federal de Goias y Universidad de Brasília
}

\author{
Geni Anastacio Sabino \\ Universidad Federal de Goias - UFG, Brasil \\ Maria Dolores Ayuso García \\ Universidad de Murcia, España
}

ANÁLISIS / ANALYSIS

\begin{abstract}
Resumen
Analiza sobre la importancia de la implementación de una Política de Protección de la Propiedad Intelectual y la diseminación de esa cultura en las universidades, pues la definición de esa política actualmente es un requisito esencial para que las Universidades inviertan en investigaciones tecnológicas patentables que puedan ser transferidas para el sector productivo. La cuestión y la discusión sobre la propiedad intelectual ha movilizado un número cada vez mayor de sectores de la sociedad brasileña donde la capitalización del conocimiento también viene volviéndose la base para el desarrollo económico y social. EI artículo presenta una investigación realizada en el ámbito de la Universidad Federal de Goiás (UFG) y Universidad de Brasilia (UnB)-Brasil, a través de un análisis comparativo buscó testar que la existencia de una Política Institucional de Protección a la Propiedad Intelectual resulta en un mayor número de registro de patentes por parte de las universidades. Participaron de la encuesta profesores/investigadores de los diversos Institutos, Facultades y Escuelas de las dos Universidades.
\end{abstract}

Palabras clave

Propiedad intelectual; Universidades públicas; Universidad Federal de Goias; Universidad de Brasília; Brasil

\section{Intellectual property brazilian universities: Federal University of Goias and Brasilia University}

\begin{abstract}
This study is about the importance of having a intellectual property protection policy and also the spread of it among the college environment of the Federal University of Goiás- Brazil once this definition is essential so that the universities invest in technology researches can be transferred to the productive area. This work analyzes on the importance of the implementation of a Protection politics of the Intellectual Property and the dissemination of this culture in the universities, since the definition of this politics at present is an essential requisite so that the Universities are invested in technological investigations by patentables that could be transferred for the productive sector. The discussion about intelectual property has been increasing the numbers of sector in Brazilian society where the knowlegde has been the principal point to the economic and social development. The dissertation presents the investigation realized in the ambience of the Federal University of Goiás (UFG) and University of Brasilia (UnB) Brazil, across a comparative analysis it thought about how to test that the existence of an Institutional Politics of Protection to the Intellectual Property results in a major number of record of patents on the part of the universities. They informed of the poll teachers / investigators of the diverse Institutes, Faculties and Schools of two Universities.
\end{abstract}

\section{Keywords}

Intelectual property ; Brazilian Public Universities ; Federal University of Goiás ; University of Brasilia ; Brasil 


\section{Introdución}

Entende-se por propiedad intelectual como una expresión genérica que trata de garantizar a inventores o responsables por cualquier producción del intelecto (ya sea en el dominio industrial, científico, literario o artístico) el derecho de conseguir, al menos por un determinado período de tiempo, recompensa por la propia creación.

De acuerdo con Sherwood (1992) la propiedad intelectual es el conjunto de dos cosas:

a) Primero, son las ideas, invenciones y expresión creativa, que son esencialmente el resultado de la actividad privada;

b) En segundo lugar hay el deseo del público de dar el status de propiedad a esas invenciones y expresiones.

La Propiedad Intelectual abraza dos grandes áreas:

- Propiedad Industrial: Patentes, Marcas, Diseños Industriales, Indicaciones Geográficas y Protección de Cultivos.

- Derecho de Autor: Obras Literarias y Artísticas, Programas del Ordenador y Dominios de Internet.

Una de las finalidades de la protección de la creación intelectual es que esta es una herramienta fundamental para el incentivo del progreso tecnológico, propiciando ventajas para la sociedad mundial.

Aunque la actividad inventiva acompañe la evolución de la humanidad desde el principio, la protección de las creaciones intelectuales remonta a momentos más recientes de la historia.

La propiedad industrial fue descrita por Mittelbach (1998) como la rama de la propiedad intelectual que aprueba la concesión de patentes, para proteger el conocimiento tecnológico, a la protección y creación ornamentales por medio de concesión de registro de diseño industrial, a la concesión de marcas, a la representación a falsas indicaciones geográficas, y a la represión de la concurrencia desleal. Toda esa materia está regulada por la Ley 9.279 del 14 de mayo de 1996, usualmente denominada Ley de Patentes.

En las décadas de 80 y 90 hubo un creciente aumento en el desarrollo científico y tecnológico, acompañado de la reducción del tiempo requerido para que el desarrollo tecnológico se incorpore a los resultados del proceso productivo; la reducción del ciclo de vida de los productos en el mercado; la elevación de los costes de investigación y desarrollo y de los riesgos implícitos en la opción tecnológica, esta enumeración de factores creó una inestabilidad que generó en una mayor preocupación respecto a la importancia de la protección a la propiedad intelectual como mecanismo de garantía de los derechos y de estímulo a las inversiones.

De acuerdo con Theotonio (1991) los nuevos paradigmas impuestos por una economía global, muestran que la innovación tecnológica surgió como factor de alta relevancia en la agregación de valor a productos y servicios. Se constató que la empresa es el principal agente catalizador de las innovaciones, pasando a ser la responsable para su comercialización. En ese contexto, cada vez más la producción industrial se tornó dependiente de una base tecnológica que evoluciona rápidamente. Además, la intensa concurrencia internacional redujo drásticamente el lapso temporal entre invención e innovación. Ante ese nuevo contexto, la innovación aparece como uno de los hilos conductores para la transferencia de tecnología de las instituciones de enseñanza e investigación. Sin embargo, las instituciones de enseñanza superior que generan conocimiento y tecnología deben considerarse beneficiarias de retorno económico y financiero de sus investigaciones en alguna medida.

Existen varios medios de proteger legalmente el conocimiento, entre ellos, la patente. Esta se define como una innovación tecnológica con aplicación industrial o comercial. Se configura como un monopolio temporal obtenido por medio del derecho exclusivo de explorar un invento concedido por el Estado y que ha recibido, específicamente, atención de las Universidades y del Gobierno sobre su papel y su importancia en las Instituciones Públicas de Enseñanza e Investigación. La intensidad del proceso de transformación del conocimiento generado en la 
Universidad en productos y procesos que beneficien a la sociedad depende de la Política de Protección del conocimiento vigente en esa Universidad.

Sin embargo, la discusión sobre la protección de la propiedad intelectual y la transferencia de tecnología todavía es un aspecto que tiene que ser analizado en muchas Instituciones de Enseñanza Superior Brasileñas, de carácter público y privado. Es importante que las Universidades discutan e implementen instrumentos legales de protección capaces de administrar internamente, de forma adecuada, la cuestión de los derechos de propiedad intelectual y la transferencia de tecnología, haciendo de esta forma compatible su misión principal con una interacción efectiva con el mundo empresarial. Lo que se ha dado en denominar, Ciencia-Tecnología-Sociedad.

Las políticas de patentes de las Universidades determinan la actitud de la administración y de su cuerpo docente en relación a las formas de transferencia de los resultados de la investigación para la sociedad. Un examen de las políticas formales y de los procedimientos y prácticas informales de una universidad puede revelar el grado de sofisticación en el análisis de la Propiedad Intelectual y la transferencia tecnológica (Matkin, 1990).

Este artículo presenta una revisión bibliográfica sobre la innovación y la propiedad intelectual en las universidades públicas brasileñas y un análisis comparativa entre la Universidad Federal de Goiás y la Universidad de Brasilia, resultado de la tesis doctoral sobre la política de protección a la propiedad intelectual.

\section{Marco conceptual}

La política científica y tecnología se realiza a través de planes temporales que son revisados en función de los recursos y las necesidades de cada país. En cada plan figura un presupuesto dividido en partidas según la asignación para ciencia básica y tecnología, una selección de prioridad y una política de personal (claves de la política científica), además de la necesaria evaluación de la productividad científica y tecnología. Los presupuestos generales de Estado son los que determinan la política científica. La partida correspondiente a I+D está condicionada por las necesidades de cada país. En líneas generales, hay una cierta tendencia a considerar que un país desarrollado debe acercarse al 3\% del PIB en inversión para la investigación tecnológica. Además del Estado hay otros mecanismos de financiación de la investigación científica y tecnología, sobre todo lo que provienen de la inversión industrial [Primo Yuffera, 199:174].

Innovar es más que crear algo nuevo. Es más que inventar. Un invento es una idea, un bosquejo o un modelo para un dispositivo, producto, proceso o sistema nuevo o perfeccionado [Viotti et al., 1997:28].

Ayuso García y Ayuso Sánchez [2005:52] afirman que para comprender la innovación tecnológica y maximizar las consecuencias que tiene sobre el crecimiento económico y el bienestar social, es necesario disponer de datos, cuantificar la innovación a partir de estadísticas nacionales comparables, lo que permite evaluar las políticas de fomento de innovación y mejorar sistemáticamente las mismas.

Freeman [1975] afirma que las primeras intervenciones acometidas por las administraciones públicas en materia de innovación fueron políticas de investigación y tecnología, dirigiéndose los esfuerzos a fomentar dichas actividades. Las medidas iniciales se diseñaron bajo una concepción lineal del proceso de generación de innovaciones, según la cual éste respondía a una secuencia ordenada de etapas desde la dotación de recursos a la investigación y el desarrollo hasta la obtención de resultados.

La Innovación como fenómeno económico, es reciente, surgiendo al final de la década de 50, pues antes la tecnología era tratada como resultado del crecimiento autónomo de la ciencia, de las artes industriales o de las ideas geniales. Antes de la década de 50, el economista Schumpeter fue el único en resaltar la importancia de la Innovación bajo la actividad emprendedora, inversiones productivas y mercado.

A partir del siglo XX, tanto los países desarrollados como los que están en desarrollo señalan la importancia de la Ciencia y de la Tecnología (C\&t) para el desarrollo del País. La innovación tecnológica asume un importante papel frente al desarrollo económico, político y social de cualquier nación. 
Las actividades de Innovación contribuyen al perfeccionamiento de nuevos productos y servicios. En ese sentido, Viotti [2003:9], señala que la Innovación tecnológica:

[...] "es la clave para el crecimiento, la competitividad y el desarrollo de empresas, industrias, regiones y países. También tiene importancia fundamental en la determinación del estilo de desarrollo de las regiones o naciones y en la forma como afecta en el presente, y afectará en el futuro, la cualidad de vida de la población en general y de sus segmentos."

La Innovación tecnológica envuelve aspectos ligados a la viabilidad económica, ya que aquel producto necesita ser comercializado a un precio capaz de remunerar la inversión hecha por la empresa que lo desarrolló.

Esta además ocurre a través de la unión de diversos factores autónomos e interdependientes que conviene destacar en este punto:

a) las investigaciones desarrolladas por los docentes en las universidades;

b) investigaciones desarrolladas por ingenieros en Instituciones Tecnológicas;

c) investigaciones desarrolladas por las empresas en el área de producción.

Ayuso García y Ayuso Sánchez [2008:39] señalan un sistema de innovación como:

"El conjunto de elementos que en el ámbito nacional, regional o local, actúan e interaccionan, tanto a favor como en contra, de cualquier proceso de creación, difusión o uso de conocimiento económicamente útil" [Fundación COTEC 2003:18].

Las autoras indican que el concepto de sistema nacional de innovación descansa sobre el entendimiento de todo proceso de innovación y su repercusión en las actividades tecnológicas y las relaciones entre los actores implicados en el Sistema: empresas, investigadores, centros oficiales de investigación públicos o privados, etc.

Para finalizar estos aspectos conceptuales, recordar las palabras de Ayuso García y Ayuso Sánchez cuando afirman que los sistemas nacionales de información determinan el valor del aprendizaje tecnológico y contribuyen a generar el cambio de un país [2003:p. 40 ].

En Brasil las políticas sobre estos aspectos de I+D, fueron elaboradas paulatinamente, y la política de ciencia y tecnología (C\&T) fue reglamentada en las última décadas del siglo XX [Schwartzman et al.,1995].

La Innovación fue más incentivada en la década de los 90 donde fueron realizados estudios teóricos que se editaron en la publicación del Libro Verde, cuyos objetivos fueron focalizados como el cambio de las políticas de C\&T, comercial e industrial de Brasil.

\subsection{La propiedad intelectual en las universidades públicas brasileñas}

La protección legal de los resultados de las investigaciones académicas en Brasil tuvo su inicio en la década del 80, debido a que el objetivo prioritario de las Instituciones de Enseñanza Superior era la formación de profesionales cualificados, y la generación de conocimientos y su libre disponibilidad bajo la forma de artículos científicos (publicaciones), disertaciones y tesis.

Si lo comparamos con los países desarrollados, el sistema universitario brasileño es bastante reciente; a partir de la década de 1970 la actividad de investigación fue completamente introducida de forma más intensa, estando basada en el parque de post-graduación implantado desde la creación del Consejo Nacional de 
Investigación- CNPq y de la Coordinación del Perfeccionamiento del Personal de Nivel Superior - CAPES en 1951, habiendo por lo tanto entre tales eventos un lapso temporal como mínimo de dos décadas (Lahorgue y Costa, 2002).

Es importante señalar que para promover la diseminación de los resultados de la investigación académica, es fundamental que ellos estén protegidos, y que por medio de esos mecanismos de protección y transferencia de tecnología, resulten en inversiones que sean revertidas a su vez, a la investigación universitaria.

Si los resultados de una investigación son publicados sin una protección, cualquier empresa podrá beneficiarse de los recursos, muchas veces públicos, en ella invertidos sin traer ningún retorno para la universidad. Córdoba (1996:125) afirma que si la Universidad pierde la protección por patente debido a una pre-publicación, esos resultados difícilmente van a interesar a la industria. Especialmente grave es este peligro de pérdida en los campos de la investigación científica de alta especialización como son las nuevas tecnologías. Por lo tanto es fundamental que las Universidades y Centros de Investigación, definan una política institucional clara y bien definida sobre la protección de la propiedad intelectual que es generada en sus laboratorios, buscando un equilibrio entre las necesidades de varios actores implicados: los profesores, los investigadores, los departamentos, la institución, las fuentes de financiamiento.

El reducido interés de los docentes tanto por la utilización económica de sus investigaciones como por la cooperación con empresas en proyectos de desarrollo conjunto, y aún en menor grado por la protección patentaría bien como de los demás instrumentos de propiedad intelectual, se debe al hecho de que tales actividades no eran indicadores importantes de la evaluación de su trabajo, teniendo esos factores poco o casi ningún mérito comparados a los tradicionales criterios de validación académica, que son compuestos por la publicación de artículos científicos, por la dirección de tesis y disertaciones y también por la participación en consejos departamentales y demás instancias decisorias de las universidades [Fujino y Stal y Plonsky, 1999].

Chamas [2002:10] destaca que varios factores pueden haber influido en el interés de los investigadores para proteger sus investigaciones. En primer lugar, hubo un aumento del flujo de información resultante del:

a) aumento del intercambio de investigadores brasileños en instituciones del exterior realizando capacitación de doctorado y doctorado integral;

b) difusión en Brasil de experiencias bien sucedidas de administración de patentes por parte de universidades e institutos de enseñanza e investigación; c) difusión vía Internet, promoviendo el intercambio de informaciones entre institutos;

d) aumento del volumen de trabajos publicados relatando la problemática de la protección y exploración de derechos de propiedad intelectual en las instituciones académicas;

e) de la propia discusión acerca de la nueva ley de patentes en la primera mitad de la década de 1990.

Estas informaciones despertaron y ampliaron el interés de los investigadores académicos en cuestiones de políticas de ciencia y tecnología, llevándolos a debatir sobre el hecho de que parte de los resultados de sus investigaciones realizadas con recursos públicos no está retornando en forma de beneficios para la sociedad.

Aunque según la autora existe un conjunto de factores que podemos llamar estructurales:

madurez de la investigación nacional en áreas que generan conocimientos tecnológicos, como es el caso de la biotecnología;

búsqueda de fuentes alternativas de recursos para la investigación;

En Brasil el Instituto Nacional de Propiedad Industrial (INPI) es la institución responsable por la protección de la propiedad industrial brasileña. En contrapartida a esta tradición, la cultura de la Propiedad Intelectual está poco desarrollada, en particular por las micro y pequeñas empresas, que hacen poco uso del INPI (Instituto Nacional de Propiedad Industrial) siendo las empresas de grandes dimensiones y las multinacionales las mayores usuarias del sistema de protección de marcas y patentes en el país, lo que es incompatible con el desarrollo nacional, ya que las micro y pequeñas empresas tienen un papel importante en la economía brasileña.

Cuando se habla de Propiedad Industrial, se habla de toda la protección de los activos intangibles que las empresas desarrollan a lo largo del tiempo, en particular, del conocimiento que son capaces de generar y de las 
marcas que, de alguna manera, consolidan el compromiso, en la mente del consumidor, de las empresas con la calidad, el desarrollo tecnológico y la innovación. La contribución del Instituto Nacional de Propiedad Industrial (INPI) es esencial para la gestión de la Propiedad Industrial y para que el sector empresarial brasileño pueda desarrollar y aumentar la competitividad.

La Protección de la Propiedad Intelectual brasileña, actualmente, se refiere a los derechos de propiedad industrial (Ley 9.279/96), y los derechos autorales (Ley 9.610/1998) y de Programas del Ordenador (Ley 9.609 de 1998) y la Ley de Protección de Cultivos (Ley 9.456 de 1997)

\subsection{Evolución de los depósitos de patentes universitarias brasileñas}

La relevancia de estudiarse la actividad de patentes de las universidades brasileñas está ligada al hecho de que, en la lista de los 20 mayores depositarios de patentes en Brasil entre 1990 y 2000, aparecen tres universidades (Universidad Estadual de Campinas (UNICAMP), Universidad de São Paulo (USP) y Universidad Federal de Minas Gerais (UFMG) y dos instituciones de investigaciones Empresa Brasileira de Pesquisa Agropecuaria (EMBRAPA) y Fundação Oswaldo Cruz (Fiocruz) [Albuquerque 2003:52].

De acuerdo con el Instituto Nacional de Propiedad Industrial (INPI) en el período de 2000 a 2004, fueron depositados 784 solicitudes de patentes universitarias, lo que representa, en apenas 5 años, un crecimiento de $130 \%$ en relación a la década de 90 . Varios aspectos contribuyeron para que el número de patentes tuviese un aumento en ese período entre ellos:

a) Acción de las Fundaciones de Amparo a la Investigación (FAPs);

b) La creación de los Núcleos de Innovación Tecnológica (NITs);

c) La posibilidad de proteger medicamentos, alimentos y productos químicos a través de la Ley no 9.279/96.

Varios factores fueron suscitados por la investigación realizada por el Instituto Nacional de Propiedad Industrial (INPI) entre ellos se puede citar:

2000-2004, la región sureste aparece con 79\% de los depósitos de patentes universitarias, destacando la Universidad de Campinas (UNICAMP), que presentó 232 depósitos, o casi 30\% del total de esa región;

Apenas 25 instituciones poseen tres o más depósitos de patentes en cinco años;

Las investigaciones se concentran en pocas áreas del conocimiento;

$81 \%$ de las solicitudes de patentes posee um único titular;

Pequeño número de sociedades entre las instituciones y los investigadores.

Es necesario crear acciones dirigidas a:

- Creación de políticas de estímulos para combatir las distorsiones regionales;

- Inversiones en sociedades entre Instituciones de Ensenãnza;

- Cruzamiento de datos entre la demanda de tecnología extranjera por las empresas brasileñas y las investigaciones nacionales.

- Inversiones em sociedades entre Instituciones de Enseñanza. 
Tabla 1 - Depósitos de las Patentes Universitarias Brasileñas de las Regiones Brasileñas en el Instituto Nacional de Propiedad Industria (INPI) en el período de 2000 a 2004

\begin{tabular}{|c|c|c|}
\hline Regiones & Total de los Depósitos & $\%$ \\
\hline Sureste & 626 & 78,90 \\
\hline Sur & 99 & 12,54 \\
\hline Noroeste & 26 & 3,30 \\
\hline Norte & 14 & 1,77 \\
\hline Centro Oeste & 25 & 3,17 \\
\hline Total & 790 & 99,68 \\
\hline
\end{tabular}

Fuente: Sinpi/Cedin/Sistema Ad. - Instituto Nacional de Propriedade Intelectual - INPI, 2007

Gráfico 1- Depósitos de Patentes de las Principales Universidades Brasileñas Período, 2000-2004

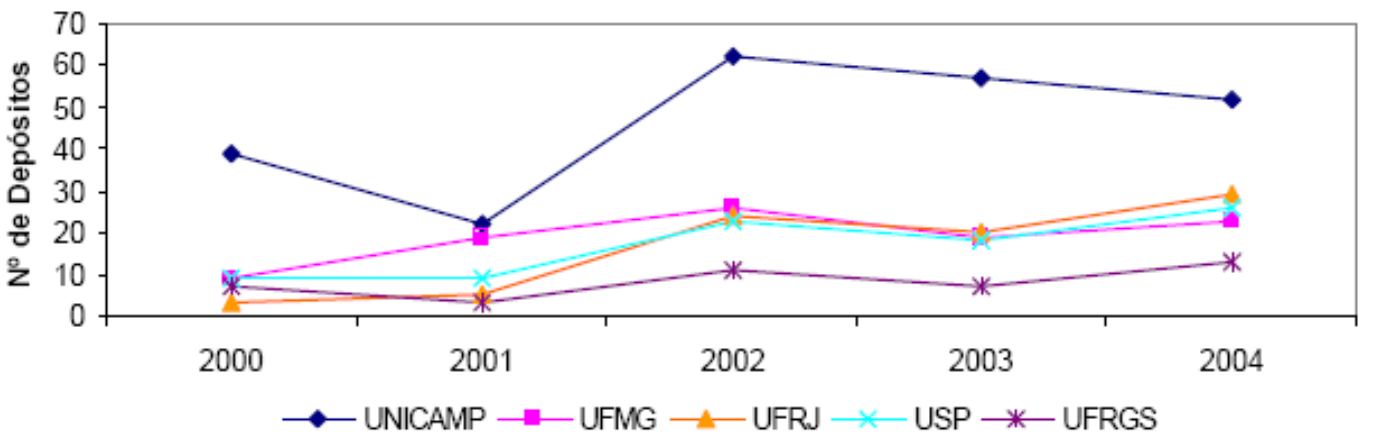

Fuente: Sinpi/Cedin/Relatórios do Instituto Nacional de Propriedade Industrial - INPI, 2007

Se observa que en el período de 2000 a 2004 hubo un considerable aumento de un 120\% en el número de depósito de patentes efectuados por las Instituciones de Enseñanza Superior, aunque de forma general la Propiedad Intelectual se encuentra diseminada en el medio académico del país. De acuerdo con datos del Instituto Nacional de Propiedad Industrial (INPI) este hecho es aún más preocupante cuando se contabiliza la totalidad de recursos invertidos en las investigaciones efectuadas por las universidades públicas, sin que haya mayor preocupación con la búsqueda por protección de estos desarrollos a través de patentes, así como su transferencia para el mercado y uso por la sociedad.

\subsection{Cooperación universidad-empresa}

La aproximación entre universidades y empresas no es tarea sencilla, teniendo en cuenta la existencia de significativas diferencias culturales que las caracterizan, empezando por la división histórica entre la misión de cada una de esas instituciones, hecho reflejado en los métodos de trabajo y en el énfasis concedido a la libertad de investigación, lo que naturalmente da origen a factores de conflicto que tienden a dificultar tal interacción [Geisler y Rubenstein, 1989].

En Brasil las actividades de investigación están desarrolladas casi exclusivamente dentro de las Universidades y Centros de Investigación Públicos (que cuentan con recursos públicos), los cuales poseen una producción científica y tecnológica abundante y que raramente llega al mercado. Las indústrias están cada vez más 
necesitadas de innovación, para estar insertas en un mercado cada vez más competitivo, infelizmente no siempre cuentan con personal especializado en investigación y desarrollo, por eso acuden a las Universidades, donde las sociedades público-privadas pasan a representar un papel importante.

Las universidades tienen como misión, la formación de recursos humanos y la realización de investigaciones de carácter eminentemente experimental, que complementan esa tarea y aumentan el nivel general de conocimientos disponibles para la sociedad. Las universidades buscan la cualidad de las investigaciones y la amplia divulgación de los resultados obtenidos. Las empresas miran el lucro y, para tal, seleccionan cuidadosamente los proyectos en los cuales deberán comprometerse, según su potencialidad comercial, riesgo y retorno económicofinanciero. Todas las informaciones relevantes son cuidadosamente resguardadas de sus oponentes. De esta forma, la empresa, en oposición a la universidad, es cerrada con relación a informaciones y estrictamente selectiva con relación a su utilización [Pavitt, 1993 apud Vedovello, 1997].

Uno de los principales obstáculos reside en el hecho de que los plazos fijados por la universidad son mayores que aquellos que le interesan a la industria y por eso habría una incompatibilidad de objetivos para el desarrollo de acciones conjuntas. Por otra parte la cooperación entre ambas instituciones es incuestionable y participan de instrumentos de cooperación [Torkomian, 1997].

En el ámbito de las políticas gubernamentales, la ausencia de una política específica tendente a estimular tal sociedad ha sido la mayor traba para la transferencia de tecnología a la industria. En el caso de la legislación, hay opiniones diferentes entre las instituciones académicas y de investigación, sobre la actual Ley de Propiedad Intelectual, específicamente en los aspectos que se refieren a la titularidad, y sus conexiones con la Ley 8.666, que trata de la obligatoriedad de licitación cuando hay interés de institución pública en procesos de compra/venda de activos [Fujino y Stal, 2004].

La cooperación es una fuente de conocimientos fluida donde los académicos pueden aplicar sus teorías a la práctica, y con eso mejorar la enseñanza, además de traer una cierta flexibilidad presupuestaria para la universidad en la compra de instrumental, infra-estructura, etc.

\subsection{Principales características de las oficinas universitarias de transferencia de tecnología}

Las oficinas de propiedad intelectual fueron creadas con la finalidad de gerenciar la transferencia de tecnología de las universidades para las empresas. En relación a la estructura organizativa, pueden ser internos dentro de la estructura administrativa de las universidades, y externos, que pueden ser en empresas o en las agencias de fomento a la investigación.

Las actividades que pueden ser desarrolladas por esas oficinas de transferencia de tecnología son:

- Consultorias;

- Investigación Contratada;

- Los Proyectos Contratados del tipo: cursos, prestación de servicios, las incubadoras y parques tecnológicos.

Santos y Rossi (2002) realizan una investigación de la situación de las universidades. Se solicitó información a 143 universidades brasileñas sobre la existencia de Oficinas de Asesoría Tecnológica. El retorno obtenido fue de apenas el 18\% de los cuestionarios (26 respuestas), siendo identificadas 25 oficinas de asesoría tecnológica o estructuras equivalentes, en funcionamiento en las universidades brasileñas. En él cuadro 12 aparece la sigla de la universidad, el nombre de la oficina, la sigla del mismo y la fecha en que fue creado: 
Tabla 2 - Relación de las Oficinas de Transferencia de Tecnología Universitarias

\begin{tabular}{|c|c|c|c|}
\hline Universidade/Sigla & $\begin{array}{l}\text { Nombre de la } \\
\text { Oficina/Sigla }\end{array}$ & Siglas & $\begin{array}{l}\text { Fecha de } \\
\text { Implantación }\end{array}$ \\
\hline $\begin{array}{l}\text { Universidad Federal de Rio Grande do } \\
\text { Sul }\end{array}$ & $\begin{array}{l}\text { Oficina de Interacción y } \\
\text { Transferencia de } \\
\text { Tecnología /EITT - }\end{array}$ & EITT & $03 / 03 / 1997$ \\
\hline Universidad do Vale do Rio dos Sinos & $\begin{array}{l}\text { Oficina de Gestión y } \\
\text { Tecnología }\end{array}$ & EGT & $18 / 09 / 1997$ \\
\hline $\begin{array}{l}\text { Pontifica Universidad Católica do Rio } \\
\text { Grande do Sul }\end{array}$ & $\begin{array}{l}\text { Agencia de Gestión } \\
\text { Tecnológica y de } \\
\text { Propiedad Intelectual }\end{array}$ & AGT & $13 / 09 / 1999$ \\
\hline FEVALE - & $\begin{array}{l}\text { Bureau de Innovación y } \\
\text { Transferencia de } \\
\text { Tecnología }\end{array}$ & BITT & $01 / 03 / 20002$ \\
\hline $\begin{array}{l}\text { Universidad Federal de Santa Maria } \\
\text { UFSM }\end{array}$ & $\begin{array}{l}\text { Núcleo de Propiedad } \\
\text { Intelectual }\end{array}$ & $\mathrm{NPI}$ & $05 / 03 / 2001$ \\
\hline UNISC & $\begin{array}{l}\text { Oficina de Transferencia } \\
\text { de Tecnología }\end{array}$ & $\begin{array}{l}\text { CPPCo } \\
\mathrm{n}\end{array}$ & $24 / 05 / 2001$ \\
\hline UCPEL & $\begin{array}{l}\text { Núcleo de Apoyo a los } \\
\text { Proyectos de Informática }\end{array}$ & NAPI & $19 / 12 / 1991$ \\
\hline UFSC & $\begin{array}{l}\text { Coordinaduría de } \\
\text { Gestión de Propiedad } \\
\text { Intelectual }\end{array}$ & $\begin{array}{l}\text { COGEP } \\
\text { I }\end{array}$ & $25 / 06 / 2002$ \\
\hline UNIOESTE & $\begin{array}{l}\text { Núcleo de Innovaciones } \\
\text { Tecnológicas }\end{array}$ & NIT & $01 / 03 / 1991$ \\
\hline UEL & $\begin{array}{l}\text { División de Innovación y } \\
\text { Transferencia de } \\
\text { Tecnología }\end{array}$ & DITT & 2000 \\
\hline Universidad de São Paulo - USP - & $\begin{array}{l}\text { Grupo de Asesoramiento } \\
\text { de Desarrollo de } \\
\text { Invenciones - sector } \\
\text { dentro de la } \\
\text { Coordinaduría Ejecutiva } \\
\text { de Cooperación } \\
\text { Universitaria y de } \\
\text { Actividades Especiales } \\
\text { (CECAE) }\end{array}$ & GAD & $24 /{ }^{\prime} 0 / ' 986$ \\
\hline $\begin{array}{l}\text { Universidad Federal de São Paulo } \\
\text { UNIFESP }\end{array}$ & $\begin{array}{l}\text { Comisión de } \\
\text { Marketing/Núcleo de la } \\
\text { Propiedad Intelectual }\end{array}$ & $\begin{array}{l}\mathrm{CMI} / \mathrm{NU} \\
\mathrm{Pl}\end{array}$ & $03 / 05 / 2000$ \\
\hline Universidad de Campinas UNICAMP & $\begin{array}{l}\text { Agencia de Innovación - } \\
\text { antes Oficina de Difusión } \\
\text { y Servicios Tecnológicos }\end{array}$ & $\begin{array}{l}\text { INOVA } \\
\text { EDISTE } \\
\text { C } \\
\end{array}$ & $28 / 08 / 1990$ \\
\hline $\begin{array}{l}\text { Universidad Federal de São Carlos - } \\
\text { UFSCAR }\end{array}$ & $\begin{array}{l}\text { Núcleo de Extensión } \\
\text { UFSCAR-Empresa }\end{array}$ & NUEMP & $01 / 019 / 1996$ \\
\hline UNIVAP & $\begin{array}{l}\text { Pro-Rectoría de } \\
\text { Integración Universidad- } \\
\text { Sociedad }\end{array}$ & $\begin{array}{l}\text { UNIVA } \\
\text { P- } \\
\text { PRIUS }\end{array}$ & $02 / 12 / 1992$ \\
\hline Universidad Federal Fluminense UFF & $\begin{array}{l}\text { Oficina de Transferencia } \\
\text { de Conocimiento }\end{array}$ & ETCO & $30 / 07 / 2001$ \\
\hline $\begin{array}{l}\text { Universidad Federal do Rio de Janeiro } \\
\text { - UFRJ }\end{array}$ & $\begin{array}{l}\text { Oficina de Transferencia } \\
\text { del Instituto Politécnico }\end{array}$ & ETT & $23 / 09 / 1996$ \\
\hline $\begin{array}{l}\text { Pontifícia Universidad Católica do Rio } \\
\text { de }\end{array}$ & $\begin{array}{l}\text { Oficina de Desarrollo del } \\
\text { Centro }\end{array}$ & $\begin{array}{l}\text { ED do } \\
\text { CTC }\end{array}$ & $03 / 01 / 1994$ \\
\hline
\end{tabular}

Fuente: Santos, M. E. R. y Rossi, A. L. (2002). Prometo Estímulo à criação e consolidação de Núcleos de Propriedade Intelectual eTransferência de Tecnologia em Universidades Brasileiras: relatório final da etapa de mapeamento das Instituições. Porto Alegre: UFRGS. 
En Brasil la Oficina de Transferencia de Tecnología, debe facilitar, incentivar y promover la interacción universidad/empresa/gobierno. Sin embargo, no existe ley definiendo y delimitando la transferencia de tecnología por las instituciones públicas brasileñas, además de faltar directrices generales y apoyo directo del Gobierno. Cada universidad ha tomado sus iniciativas aisladamente. De acuerdo con la investigación realizada, los servicios prestados por las oficinas no son semejantes entre ellos, agrupando todos los servicios en una lista, se obtienen: atención a demandas tecnológicas, gestión de servicios tecnológicos, negociación de proyectos, elaboración de convenios y contratos, registro de la propiedad intelectual, comercialización de tecnologías y patentes, capacitación de recursos humanos y promoción de eventos de difusión tecnológica. La estructura organizativa de las oficinas es variada: la mayoría (72\%) no posee dotación presupuestaria propia; el 28\% generan recursos propios; la estructura no es centralizada en un $60 \%$, es centralizada en el $20 \%$ y en el $20 \%$ es centralizada apenas en parte; la mayoría (76\%) usa una fundación de apoyo para desarrollar sus actividades; el $52 \%$ utilizan mecanismos de acompañamiento de proyectos [Santos y Rossi, 2002].

Transferir conocimiento, procesos y tecnología es permitir el desarrollo sustentable del país. Sin embargo, la universidad aún no sabe transferir conocimientos. Y para eso, ella necesita aprender a aprender a negociar. Hoy muchas instituciones están dando un paso significativo en esa dirección, cuando se preocupan en aprender a proteger sus inventos. Con la creación de Oficinas de Transferencia de Tecnología, la universidad está ofreciendo las condiciones básicas para trabajar la cuestión de la negociación de aquello que ya fue o será protegido. Está invirtiendo en la formación de profesionales en negociación para diseminar y decir a los investigadores que ellos no sólo deben proteger sus inventos, sino además negociarlos. Investigadores, normalmente no son profesionales de negociación. Esa es una cuestión que normalmente transciende sus intereses. Profesionalizar la diseminación de conocimientos y de tecnologías, puede representar a la universidad pública un salto cualitativo en sus relaciones, porque aunque tiene el conocimiento, lo que falta es una transferencia disciplinada y objetiva [Macedo, 2000].

\subsection{Contextualización de las Universidades: Federal de Goiás y Universidad de Brasília}

La Universidad Federal de Goiás-UFG, Institución Federal de Enseñanza e Investigación de nivel superior vinculada al Ministerio de Educación y del Deporte. Se creó el 14 de diciembre de 1960, con la reunión de cinco Escuelas Superiores ya existentes en Goiânia: la Facultad de Derecho, la Facultad de Farmacia y Odontología, que hoy forman dos unidades distintas, la Escuela de Ingeniería, que a partir de 1991 fue dividida en dos unidades, Escuela de Ingeniería Civil y Escuela de Ingeniería Eléctrica, el Conservatorio de Música y la Facultad de Medicina. En 1963, atendiendo a las demandas del sector agropecuario del Estado, se funda la Escuela de Agronomía y Veterinaria, que actualmente constituyen dos unidades distintas. En 1964, el Instituto de Matemática y Física inicia sus actividades. Con la reforma universitaria de 1968, la Facultad de Filosofía se divide dando origen al Instituto de Ciencias Humanas y Letras, al Instituto de Química y Geociencias, al Instituto de Ciencias Biológicas y a la Facultad de Educación. También se crea el Instituto de Artes, como resultado de la fusión del Conservatorio de Música y de la Facultad de Artes. Posteriormente, serían creadas otras dos unidades en el área de la salud: el Instituto de Patología Tropical y la Facultad de Enfermería y Nutrición. Con la fundación de la Facultad de Educación Física, en 1988, la UFG Ilega a comportar 17 unidades académicas. En 1996, la Universidad fue reestructurada administrativa y académicamente, lo que resultó en la separación de tres institutos básicos - Instituto de Matemática y Física, Instituto de Ciencias Humanas y Letras, Instituto de Química y Geociencias - en ocho unidades académicas: Instituto de Matemática y Estadística, Instituto de Física, Instituto de Informática, Facultad de Comunicación y Biblioteconomía, Facultad de Ciencias Humanas y Filosofía, Facultad de Letras, Instituto de Química y Instituto de Estudios Socio-Ambientales. La Facultad de Enfermería y Nutrición fue separada en dos nuevas unidades: Facultad de Enfermería y Facultad de Nutrición, y el Instituto de Artes también siguió el ejemplo, Facultad de Artes Visuales y Escuela de Música. Las recientes reestructuraciones administrativas y académicas elevaron a 24 el total de las unidades académicas y un Centro de Enseñanza y Pesquisa Aplicado a la Educación - que suministra enseñanza primaria y secundaria. Además de los dos Campi situados en Goiânia - Campus Samambaia y Campus Colemar Natal e Silva - la UFG cuenta con otros cinco campi avanzados - Catalão, Jataí, Firminópolis, Cidade de Goiás y Rialma. 
Esta Universidad, tiene como objetivos principales la formación de profesionales altamente calificados en las varias áreas del conocimiento, la Investigación en los diversos campos académicos, la implementación de programas y proyectos de actuación en la realidad regional y nacional y la creación, promoción y difusión de innovaciones tecnológicas y de programas artísticos y culturales.

Tabla 3 - Datos Numéricos de la Universidad Federal de Goiás en 2007

\begin{tabular}{|c|c|}
\hline \multicolumn{2}{|c|}{ Estructura Administrativa } \\
\hline Profesores & Total \\
\hline Doctores & 741 \\
\hline Maestria & 429 \\
\hline Especialización & 87 \\
\hline Graduado & 37 \\
\hline Profesores Substitutos & 363 \\
\hline \multicolumn{2}{|c|}{ TOTAL 1.657} \\
\hline TécnicosAdministrativos & 1.862 \\
\hline \multicolumn{2}{|c|}{ Estructura Acadêmica } \\
\hline Cursos & Total \\
\hline Post-Graduación Stricto Sensu & 45 \\
\hline $\begin{array}{l}\text { Doctorado: Agronomía, Biología, Ciencia Animal, } \\
\text { Ciencias Ambientales, Ciencias de la Salud, } \\
\text { Educación, Geografía, Historia, Letras y } \\
\text { Lingüística, Medicina Tropical y Química. }\end{array}$ & 13 \\
\hline $\begin{array}{c}\text { Maestría: Negocios del Campo, Agronomía } \\
\text { Campus Jataí, Biología, Ciencia Animal, Ciencia } \\
\text { de la Computación, Ciencia y Tecnología de } \\
\text { Alimentos, Ciencias de la Salud, Ciencias } \\
\text { Farmacéuticas, Comunicación, Cultura Visual, } \\
\text { Derecho, Ecología y Evolución, Educación, } \\
\text { Enfermería, Ingeniería Civil, Ingeniería del Medio } \\
\text { Ambiente, Ingeniería Eléctrica y de Computación, } \\
\text { Filosofía, Física, Geografía, Geotecnia y } \\
\text { Construcción Civil, Histórica, Letras y Lingüística, } \\
\text { Matemática, Medicina Tropical, Música, } \\
\text { Odontología, Química y Sociología. Dos Cursos de } \\
\text { Maestrías múltiples institucionales, uno en Agro } \\
\text { Negocios, y otro en Ciencias de la Salud, ambos } \\
\text { con la UFMG (Universidad Federal de Minas } \\
\text { Gerais) y la UNB (Universidad de Brasilia) y un } \\
\text { doctorado en Ciencias de la Salud, también } \\
\text { múltiple-institucionales. }\end{array}$ & 32 \\
\hline Alumnos de Graduación & 14.257 \\
\hline $\begin{array}{c}\text { Alumnos de Post-Graduación Maestria y } \\
\text { Doctorado }\end{array}$ & 2.474 \\
\hline Proyectos de Investigación & 1.445 \\
\hline $\begin{array}{l}126 \text { grupos de Investigación registrados en el } \\
\text { Directorio de Grupo de Investigación del CNPq } \\
\text { (Centro Nacional de Investigación), en diferentes } \\
\text { áreas del conocimiento, como: Hipertensión } \\
\text { Arterial, Salud Bucal, Lenguas Indígenas, Estudios } \\
\text { Ambientales, Tecnología de Alimentos, } \\
\text { Mejoramientos de Plantas, Entero Toxemia Bovina, } \\
\text { Gastroenterología, Epidemiología e Inmunología, }\end{array}$ & \\
\hline
\end{tabular}




\begin{tabular}{|c|c|}
\hline $\begin{array}{c}\text { Biología Molecular, Comportamiento Político y } \\
\text { Alimentación del Trabajador. Además de esto, } \\
\text { algunos centros de Investigación se encuentran } \\
\text { consolidados en la Institución, como el Centro de } \\
\text { Tecnología de Alimentos, Resonancia } \\
\text { Paramagnética, Control Remoto, Bioquímica, } \\
\text { Farmacología, Enfermedades de Llagas, entre } \\
\text { otros. }\end{array}$ & \\
\hline \multicolumn{2}{|c|}{ Producción Científica } \\
\hline $\begin{array}{l}\text { Artículos Publicados en Anales de Eventos } \\
\qquad \text { Científicos }\end{array}$ & 5.124 \\
\hline $\begin{array}{l}\text { Artículos Publicados en Periódicos Nacionales e } \\
\text { Internacionales }\end{array}$ & 1.200 \\
\hline Publicación de Libros /Capitulos de Livros & 85 Títulos/ 200 Capitulos \\
\hline Disertaciones de Maestría y Tesis Doctorales & 676 \\
\hline Proyectos de Investigación en Ejecución & 1.073 \\
\hline Programa de Extensión Universitaria & 431 Proyectos \\
\hline $\begin{array}{c}\text { Educación a Distancia: Curso de Licenciatura en } \\
\text { Artes Visuales; Curso de Especialización en } \\
\text { Metodología de Enseñanza Fundamental;Curso de } \\
\text { Licenciatura en Física; Curso de Licenciatura en } \\
\text { Ciencias Biológicas; Curso de Administración - } \\
\text { Bachillerato }\end{array}$ & 655 \\
\hline
\end{tabular}

Fuente: Información recogida en: http://www.ufg.br. Acceso: 30-04-08

\subsection{Universidad Nacional de Brasilia (UnB)}

El 15 de diciembre de 1961, el presidente de la República João Goulart sancionó la ley 3.998, que autorizó la creación de la Universidad. El antropólogo Darcy Ribeiro, ideólogo, fundador y primer rector de la UnB, soñaba con una institución volcada en las transformaciones diferentes del modelo tradicional creado en la década de 1930. En Brasil, fue la primera Universidad en estar dividida en institutos centrales y Facultades, y desde esta perspectiva, se crearon los cursos-troncales, en los cuales los alumnos tenían su formación básica. Después de dos años, seguían para los institutos y Facultades. Los tres primeros cursos-troncales eran: Derecho, Administración y Economía, Letras Brasileñas, y Arquitetura y Urbanismo. 
Tabla 4 - Datos Numéricos de la Universidad de Brasilia en 2007

\begin{tabular}{|c|c|}
\hline \multicolumn{2}{|c|}{ Estructura Administrativa } \\
\hline Profesores & Total \\
\hline Doctores & 1.129 \\
\hline Maestria & 276 \\
\hline Especialización & 11 \\
\hline Graduación & 29 \\
\hline \multicolumn{2}{|c|}{ TOTAL 1.445} \\
\hline TécnicosAdministrativos & 2.525 \\
\hline \multicolumn{2}{|c|}{ Estructura Acadêmica } \\
\hline Cursos & Total \\
\hline \multicolumn{2}{|l|}{ Post-Graduación Stricto Sensu } \\
\hline Doctorado & 45 \\
\hline Maestría & 64 \\
\hline Especialización & 73 \\
\hline Alumnos de Graduación & 21.471 \\
\hline Alumnos de Post-Graduación & 6.327 \\
\hline \multicolumn{2}{|c|}{ Producción Científica } \\
\hline Títulos Publicados & 81 \\
\hline
\end{tabular}

Fuente: Elaboración propia a partir de los datos recogidos: http://www.UnB.br. Acceso: 29-05-08

\subsection{Núcleo de Propiedad Intelectual y Transferencia de Tecnología}

La UnB estableció, en 1998, un régimen normativo para la protección de las tecnologías innovadoras desarrolladas en esta Universidad. La resolución del Consejo de Administración transfirió la responsabilidad de la gestión de los registros de patentes al Centro de Apoyo al Desarrollo Tecnológico (CDT), que creó Núcleo de Propiedad Intelectual y Transferencia de Tecnología (Nupitec). El Núcleo de Propiedad Intelectual y Transferencia de Tecnología, NUPITEC, es responsable de la gestión de las innovaciones generadas en la Universidad de Brasilia.

\section{Servicios}

Los servicios que a continuación detallamos son los más destacados de los que en realidad realizan. Los mismos son:

1. Consultoría en propiedad intelectual para la comunidad externa vía Llame Tecnología y Servicio Brasileño de Respuestas Técnicas - SBRT;

2. Asesoría técnica en los trámites de protección y acompañamiento de las tecnologías de la Universidad de Brasilia;

3. Comercialización de las tecnologías generadas en la Universidad para el sector productivo, por medio de transferencia o licencia.

El Núcleo de Propiedad Intelectual y Transferencia de Tecnología (NUPITEC) atiende a todo público "blanco", esto es, investigadores, profesores, alumnos, técnicos administrativos y/o técnicos de laboratorios que 
hayan desarrollado productos o procesos innovadores. Al entrar en contacto con el Núcleo de Propiedad Intelectual y Transferencia de Tecnología (NUPITEC), se informa al inventor/autor de la Resolución 005/98, que prevé la distribución de las ganancias económicas, provenientes de creación intelectual en la UnB. El investigador toda vez que ha rellenado el Impreso de Identificación de su innovación y a partir de este informe, el centro realiza las búsquedas en banco de datos tecnológicos nacionales e internacionales, además de una investigación previa para comprobar el potencial de mercado, con respecto al invento presentado. Esta búsqueda retrospectiva, es en realidad un "estado de la técnica".

Todas las etapas del trabajo realizado por el Núcleo de Propiedad Intelectual y Transferencia de Tecnología (NUPITEC) son extremamente importantes. La búsqueda de antecedentes evita la duplicación de esfuerzos, esto es, investigar lo que ya fue investigado o inventado. La mayor parte de la información tecnológica más reciente existente en el mundo está en los documentos de patentes, siendo así, que una búsqueda retrospectiva bien hecha podrá garantizar que las innovaciones sean concedidas cuando sea solicitada su protección. Además, es importante proteger las innovaciones, pues además del prestigio académico, las ideas innovadoras pueden ser transformadas en retorno financiero y en desarrollo económico para el país ligado al PIB, pero también a la innovación y al desarrollo tecnológico.

Tras el análisis de potencialidad de mercado y la protección de la invención, la misma comienza un proceso para ser concedida. Por tratarse de Derechos exclusivos, las empresas que obtienen las licencias o concesión consiguen una mejor posición en el mercado, ganando tiempo y experiencia para probar y comercializar los productos. Y si el depositante está sufriendo perjuicio por concurrencia desleal de alguien que esté produciendo el mismo objeto de su invención, éste podrá contactar tal concurrencia notificándolo en el sentido de que, si el concurrente insiste en la práctica desleal él podrá, cuando obtenga la Carta-Patente, emprender una acción judicial de indemnización por pérdidas y daños, que podrán ser contabilizados a partir de la fecha de publicación de la patente. En este caso, la publicación anticipada es útil para efectos de la determinación de esta fecha. Se garantiza de esta forma la exclusividad de producción y comercialización de los bienes protegidos.

Como ocurre en las demás instituciones, las innovaciones producidas en la UnB están patentadas a nombre de la Universidad. Ahora bien, los recursos generados a partir de la comercialización del invento se dividen entre la institución y el inventor, que recibe un tercio del total de los rendimientos. Los dos tercios restantes se dividen entre la Universidad de la siguiente forma:

- El $20 \%$ se destina a las actividades de investigación y desarrollo en la Facultad a que el inventor esté vinculado.

- El $30 \%$, al departamento del inventor, destinado a las actividades de investigación y desarrollo.

- Un $20 \%$ a la unidad gestora de la propiedad intelectual.

- El $30 \%$ para la administración superior, destinado a un fondo de reserva para financiamiento de actividades de investigación y desarrollo tecnológico e industrial.

Una de las reglas seguidas por la UnB y que deseamos destacar, es que los registros de patentes siempre se hacen de forma simultánea en Brasil y en los Estados Unidos, lo que amplía notablemente su visibilidad internacional. Actualmente el Núcleo de Propiedad Intelectual y Transferencia de Tecnología (NUPITEC) posee activos intangibles protegidos, distribuidos tal y como se indica en La tabla 
Tabla 5- Número de Activos Intangibles Protegidos 1999-2007

\begin{tabular}{|c|c|}
\hline Activos Intangibles Protegidos & Cantidad \\
\hline Total Patentes UnB & 72 \\
\hline Patentes FUB & 33 \\
\hline Patentes co-titularidad & 39 \\
\hline Software & 06 \\
\hline Diseño Industrial & 01 \\
\hline Derecho Autorial & 02 \\
\hline Marcas & 44 \\
\hline Total & $\mathbf{1 2 5}$ \\
\hline
\end{tabular}

Fuente: Datos recogidos: http://www.UnB.br. Acceso: 29-05-08

\section{Metodologia y resultados}

La metodología empleada en la investigación, así mismo exponer y describir detalladamente el conjunto de tareas realizadas en la investigación y el conjunto de materiales utilizados para esta finalidad. La finalidad de la actividad científica se resume, de una forma amplia, como el intento de obtención de la verdad, la cual se realiza por medio de la comprobación de hipótesis. Asimismo, éstas son puentes entre la observación de la realidad y la teoría científica que busca explicarla.

Sierra Bravo (2003) afirma que en:

"El método de investigación científico, como en todo método, se pueden distinguir su contenido el método propiamente dicho, formado fundamentalmente por la serie de etapas sucesivas a seguir para alcanzar el resultado pretendido y su base racional, constituida por el conjunto de ideas que sirven de fundamento y de orientación al método propiamente dicho. El método, como procedimiento está constituido por las etapas generales de actuación que forman su contenido y por técnicas o procedimientos concretos, operativos para realizar en un caso determinado las fases generales de actuación en cuestión. Estas técnicas específicas de cada ciencia, pueden ser muy diversas, porque cada objeto de investigación reclama sus técnicas propias."

Este estudio está situado en el grupo de investigación exploratoria, por las características del mismo en relación al grado de novedad y porque el tema escogido es poco explorado de forma científica. 
La investigación exploratoria objetiva, en general puede provocar la aclaración de una situación para la toma de conciencia. "Un estudio exploratorio ocupa el primero de cinco niveles diferentes y sucesivos, siendo indicado [...] cuando existe poco conocimiento sobre el fenómeno" [Chizzotti, 1995:104].

Para la realización de este trabajo, se optó por la investigación cualitativa de carácter exploratoria y un análisis comparativo entre la Universidad Federal de Goiás (UFG) y la Universidad de Brasilia (UnB).

La investigación cualitativa fue subsidiada por la investigación bibliográfica, consecuencia del análisis retrospectivo de la literatura científica y búsquedas en: libros, revistas especializadas, periódicos, disertaciones y tesis. Esto es, tanto documentos primarios como secundarios o literatura gris no convencional y bases de datos tanto nacionales como internacionales. Estos materiales en su conjunto sirvieron de análisis para la formulación del cuestionario aplicado a los profesores participantes de la investigación.

El análisis estadístico utilizado en este trabajo se ha hecho siguiendo la literatura científica, en concreto, Barbetta (2001), pues el autor describe en ese método seis fases distintas que retratan el análisis cualitativo, necesario para la interpretación de los datos obtenidos en la presente investigación. El período de duración de esta investigación fue de octubre de 2007 a marzo de 2009. Para la realización de esta investigación se analizaron dos Universidades Públicas Brasileñas: La Universidad Federal de Goiás (UFG) objeto de este estudio y la Universidad de Brasilia (UnB), ambas situadas en la región centro oeste de Brasil. Los sujetos participantes de este estudio fueron los profesores de las dos Universidades Públicas Brasileña.

Para la 1a etapa de la recogida de datos fueron enviados vía correo electrónico a la Universidad Federal de Goiás (UFG) y la Universidad de Brasilia (UnB) 695 encuestas conteniendo 25 preguntas cerradas de selección múltiple y una carta de presentación del Coordinador General de Investigación y Pos-Graduación de la Universidad Federal de Goiás-Brasil, presentando la investigadora y recalcando también la importancia de este estudio para la Universidad Federal de Goiás.

La 2 $2^{a}$ etapa de la recogida de datos, contó con la participación directa de la investigadora de este trabajo, que estuvo presente en todas las Unidades Académicas de las dos Universidades participantes de esta investigación para recoger las encuestas respondidas por los profesores.

La 3a ${ }^{\text {a }}$ etapa de este estudio fue realizada a través de la investigación documental, en diversos Departamentos de las Universidades. Se consultaron y analizaron los documentos internos tales como: resoluciones, actas, informes, reglamentos. Ese análisis fue importante para ordenar aspectos gerenciales y estructurales relacionados a la protección de la propiedad intelectual en la Universidad Federal de Goiás (UFG) y en la Universidad de Brasilia (UnB).

La 4aㅡ etapa de esta investigación presenta la tabulación de los datos recogidos en cada encuesta aplicada. Entre los enviados (695), se obtuvo un total de 98 encuestas respondidas, de las que se realizaron los análisis estadísticos de acuerdo con Barbetta (2001), objeto de nuestra investigación.

Los modelos de Pzerworski (1970), los diseños del "más semejante" y del "más diferente", son formas sistemáticas de aplicar el método comparativo. De forma resumida, el diseño del "más semejante" consiste en analizar contextos bastante semejantes en los cuales una variable diferente determina la diferencia en la variable dependiente; el diseño del "más diferente", por su vez, se trata del proceso opuesto, cualquiera que sea, en contextos bastante diferentes, una variable semejante determina la semejanza en la variable dependiente.

La hipótesis que se pretende testar en este trabajo es la de que la existencia de una Política Institucional de Protección a la Propiedad Intelectual repercute en un mayor número de registro de patentes por parte de las universidades. Las universidades objeto del estudio comparado son la Universidad Federal de Goiás (UFG) y la Universidad de Brasilia (UnB).

La hipótesis causal de la tesis presenta como variable independiente principal de control la existencia de una Política Institucional de Protección a la Propiedad Intelectual. Asimismo, el número de patentes registradas por las universidades se presenta como variable dependiente.

En el análisis realizado en este trabajo se ha aplicado el modelo del diseño del "más semejante" de Pzerworski. La variable independiente anteriormente citada es considerada como variable de control pues es la 


\section{Biblios La propiedad intelectual en las universidades brasileñas}

configuración de la que es considerada determinante por la hipótesis para explicar la diferencia en la variable dependiente. Para completar el diseño, se presentaran otras dos variables independientes principales: el perfil de la universidad y el perfil de sus profesores. De esa forma, el diseño del más semejante se completa, toda vez que se supone que, para las variables principales independientes perfil de la universidad y perfil de los profesores, la Universidad Federal de Goiás (UFG) y la Universidad de Brasilia (UnB) sean semejantes, mientras que para la variable de control se imagina que ellas son diferentes. 
Tabla 6 - Hipótesis de la tesis

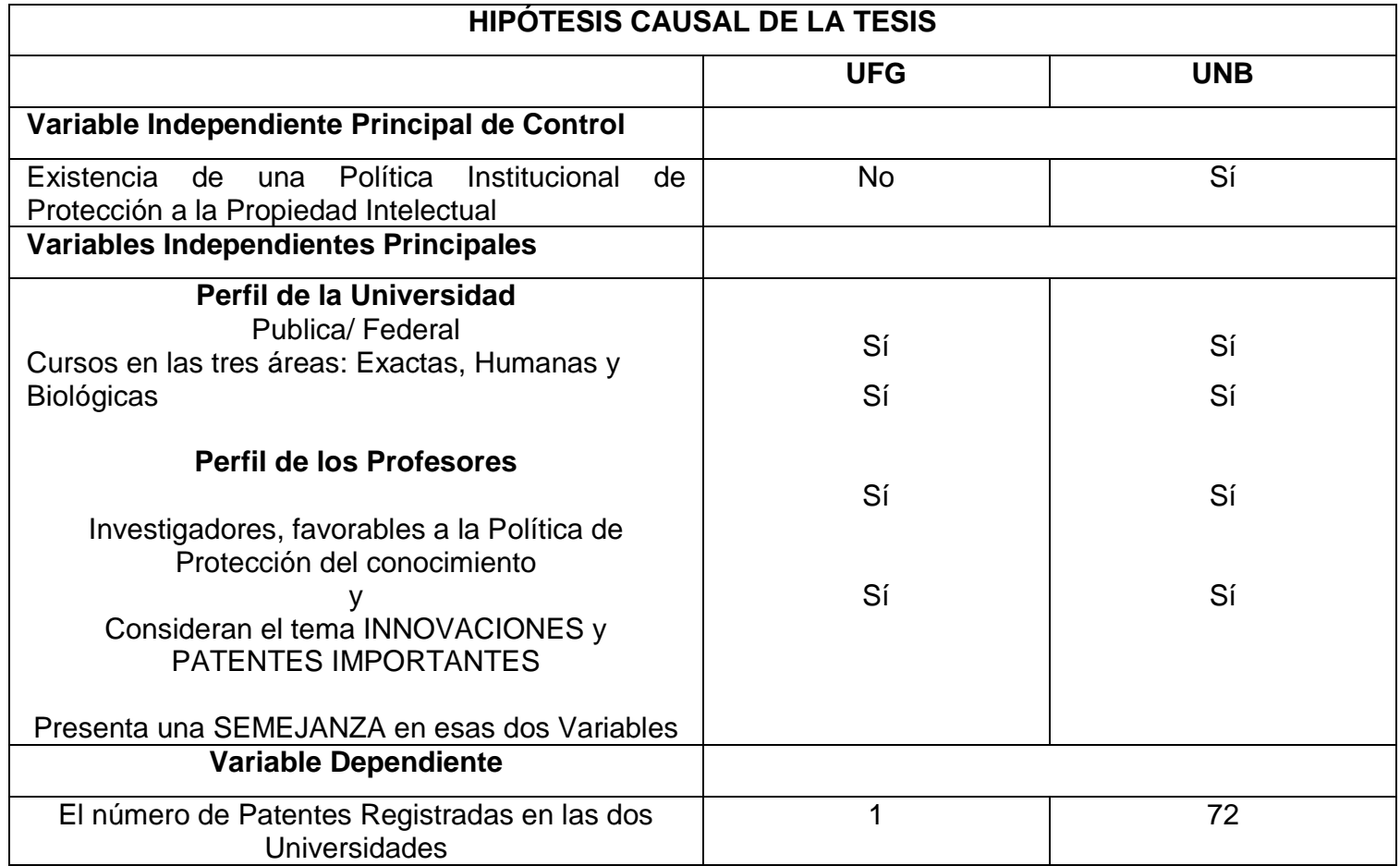

La existencia de una Política Institucional de Protección a la Propiedad Intelectual, variable independiente principal de control, se llevó a cabo de acuerdo con la observación minuciosa de los proyectos implementados por las universidades para la promoción de este tema. Se concluyó que el Núcleo de Protección de la Propiedad Intelectual de la Universidad de Brasilia (UnB) además, implementó muchos más proyectos para la promoción de la protección del conocimiento, como seminarios, palestras y cursos, que el de la Universidad Federal de Goiás (UFG).

La Universidad Federal de Goiás deberá definir una política institucional clara sobre la protección de la propiedad intelectual que produce, buscando un equilibrio entre las necesidades de varios actores envueltos, entre ellos: los departamentos, los profesores investigadores, y las fuentes de financiación, entre otros. La implementación de esa política de protección a la propiedad intelectual debe estar en consonancia con los objetivos y la misión de la Universidad, su estructura y la cualidad de las investigaciones desarrolladas, expresando de esta forma las necesidades y las especificidades de la comunidad académica.

\section{Conclusiones}

Actualmente el interés por la propiedad intelectual está creciendo de forma considerable en Brasil, pues la actual política de ciencia y tecnología brasileña (C\&T), a través de sus acciones estratégicas ha incentivado la innovación tecnológica en el medio académico y en el sector empresarial. La reformulación y actualización de la legislación de propiedad intelectual brasileña permite la adecuada protección de la invención del investigador y propicia un ambiente favorable a la inversión, por parte del sector productivo, en las investigaciones patentables dentro de las Universidades e Instituciones de Investigación.

Proteger el conocimiento generado en las Universidades exige de sus gestores decisiones volcadas para preservar su misión, que es la enseñanza, la investigación y la extensión, pero también es necesario una reflexión en torno a que la universidad necesita divulgar sus resultados de investigación para la sociedad. 
En cuanto al conjunto de las Universidades Públicas Brasileñas no tienen apenas en la actualidad formación sobretodo en la estructuración de sus Núcleos de Propiedad Intelectual. Las informaciones obtenidas como resultante de esta investigación, reflejan tales características que también denotan la ausencia de una política global que permita el crecimiento más uniforme entre regiones e instituciones de enseñanza y generación y protección del conocimiento, reflejado en el desfase de los indicadores de desarrollo del área en las Universidades.

Es importante resaltar que una política de protección de la propiedad intelectual para las Instituciones de Enseñanza Superior, puede y debe constituirse en un valioso instrumento político, en el sentido de potenciar la transferencia de tecnología entre universidades y empresas. En este sentido, la implantación de una Política de Protección de Propiedad Intelectual para la Universidad Federal de Goiás-Brasil, debe estar adecuadamente inserta en la política nacional de innovación tecnológica, con esfuerzos focalizados en la capacitación y formación de recursos humanos, pero también en la contribución al desarrollo industrial, buscando direccionar su infraestructura y esfuerzos de investigación en solucionar los problemas de la comunidad e interactuar con el sector privado.

A pesar de que la Universidad Federal de Goiás-Brasil creó en el año 2005, como determina la Ley de Innovación n 10.973 de 2004, el Núcleo de Innovación Tecnología (NIT) es necesario aún contar con diversos factores tales como: normalización interna adecuada, apoyo de la alta dirección, recursos financieros e infraestructura y principalmente de la voluntad política de la alta dirección de la Universidad, pues aún existe un largo camino por recorrer, en relación al cambio de cultura relacionada con la protección de los bienes intelectuales generados en esta Institución de Enseñanza Superior. Se espera que el esfuerzo realizado en esta investigación sirva de referencia para las Universidades Públicas y Privadas insertas en el Estado de Goiás, pero sobretodo que se implemente la Política de Protección de la Propiedad Intelectual y que estudios futuros vengan a explicitar el papel del conocimiento protegido para la inserción de tecnologías patentables en las empresas promoviendo el desarrollo regional más igualitario entre la sociedad basado en Ciencias y Tecnología (C\&T).

\section{Bibliografía y fuentes consultadas}

Albuquerque, E. (2003). Patentes e atividades inovativas: uma avaliação preliminar do caso brasileiro. En. Viotti, E. B. Macedo, M. M. (Orgs). Indicadores de ciência, tecnologia e inovação no Brasil. Campinas: UNICAMP.

Araújo, V. M. R. H. (1981). A patente como ferramenta de informação. Ciência da Informação, 10 (2), 27-32.

Assumpção, E. (2000). O sistema de patentes e as universidades brasileiras no anos 90. Brasilia: INPI/CEDIN. Disponível em http://www.geocities.com/prop industrial. Acesso em 10-10-2008.

Ayuso García, M. D.; Ayuso Sánchez, M. J. (2008). Fuentes para el estudio de la innovación. Murcia: Fundación Séneca, 206p. Disponible: www.f. seneca.org/seneca/html/inicio.htm.

Ayuso-García, M. D.; Ayuso Sánchez, M. J. (2003). Revisión de los estudios orientados a la medición de las capacidades tecnologías por medio de la literatura patente. Propuesta de análisis estadístico y evaluación de la calidad de una base de datos en patentes. Revista General de Información y Documentación, 13 (1), 151-172. 296

Ayuso García, M. D.; Ayuso Sánchez, M. J. (2002). La nueva configuración de los sistemas de ciencia y tecnología: los indicadores de innovación tecnológica en continua transformación. Investigación Bibliotecológica, 16(33):5-21.

Ayuso García, M. D.; Ayuso Sánchez, M. J. (2003). La innovación en España y en la Unión Europea. Tipología, concepto y sistemas. 1ำed. Murcia: Diego Marín librero editor.

Ayuso García, M. D.; Ayuso Sánchez, M. J. (2006). Fuentes para el estudio de la innovación. Murcia: Fundación Séneca - Agencia de Ciencia y Tecnología de la Región de Murcia. p. 22

Barbetta, Pedro A. (2001). Estatística aplicada às Ciências Sociais. 4.ed. Santa Catarina: Editora UFSC.

Caridad Sebastián, M.; Ayuso García, M.D. y Ayuso Sánchez, M.J. (2004). Política de innovación y desarrollo tecnológico em España, en el contexto del Espacio Europeo de la Investigación. Revista Interamericana de Bibliotecología. Vol.27, № 2, p. $13-47$.

Chamas, C. I. (2001). Proteção e exploração econômica de propriedade intelectual em universidades e instituições de pesquisa. 269 f. Tese (Doutorado), Universidade Federal do Rio de Janeiro, Faculdade de Ciências em Engenharia de Produção. 
Chamas, C. I. (2002). Propriedade Intelectual: uma agenda para o Mercosul. En: FÓRUM BRASIL-EUROPA Florianópolis. Anais... Rio de Janeiro: Fundação Konrad Ademauer,

Chamas, C. I. (2004). Propriedade intelectual em instituições acadêmicas: aspectos gerenciais. Revista da ABPI - Associação Brasileira da Propriedade Intelectual, 70, 3-18. Maio/Jun.

Chizzotti, A. (1995). Pesquisa em ciências humanas e sociais. 2. ed. São Paulo: Cortez.

Cordoba, S. F. (1996). Derecho de patentes e investigación. Valencia: Tirant to Blanch, Valencia.

Freeman, C. (1975). La teoria econômica de la innovación industrial. Madrid, Alianza Editorial.

Freeman, C. (1995). The National System of Innovation: in historical perspective. Cambridge Journal of Economics, $19,5-24$.

Freeman, C.; Soete, L. (1997). The economic of industrial innovation. Cambridge Massachusetts: The Mit Press, Third Edition.

Fujino, A.; Stal, E.; Plonsky, G. A. (1999). A Proteção do conhecimento na universidade. Revista de Administração São Paulo, 34 ( 4).

Fujino, A.; Stal, E. (2004). Gestão da propriedade intelectual na universidade pública brasileira: diretrizes para licenciamento e comercialização. In: Simpósio de Gestão da Inovação Tecnológica, 23, Curitiba, Anais... São Paulo:USP?PGT, 921-927.

Geisler, E. ; Rubenstein, A. H. (1989). University- Industry Relations: a review of major issues. In: A Cooperative Research and Development: The Industry - University- Government Relationship, Boston, MA, US, N. Link e G. Tassey (editors), Kluwer Academic Publishers.

Lahorgue, M. A. ; Costa, C. F. (2002, novembro). Regulação e Inovação: o caso das universidades do Rio Grande do Sul. En: Anais do XXII Simpósio de Gestão da Inovação Tecnológica - SGIT, Salvador,BA.

Macedo, M. F. G. (2000). Patentes, Pesquisa \& Desenvolvimento: um manual de propriedade industrial. Rio de Janeiro: Fiocruz, $164 \mathrm{p}$.

Macedo, M. F. G.; Barbosa, A. L. F. (2000). Patentes, pesquisa e desenvolvimento. 20.ed. Rio de Janeiro:Fiocruz.

Matkin, G.W. (1990). Technology transfer and the university. New York, American Council on Education/MacMillan Publishing Company. 329p.

Mittelbach, M. M. (1998). Propriedade Industrial. En: Cavalcanti, Ana Regina de Holanda (Coord.). Mesa Redonda Regulamentação da Propriedade intelectual no Brasil: situação atual. Rio de Janeiro: REPICT; Brasília: ABIPTI.

Póvoa, L. M. (2006). Depósitos de patentes de Universidades Brasileiras (1979-2004). Extraído el 28 de Abril, de http://www.cedeplar.ufmg.br;seminário /2006/D06AA6.pdf.

Primo Yuffera (1994). Introdución a la investigación científica y tecnologíca.Madrid, Alianza, 1994. p. 305-370

Pzerworsky, A; Teune, H. (1970). The logic of comparative social inquiry. New York: John Wiley \& Sons.

Santos, M. E. R. y Rossi, A. L. (2002). Prometo Estímulo à criação e consolidação de Núcleos de Propriedade Intelectual e Transferência de Tecnologia em Universidades Brasileiras: relatório final da etapa de mapeamento das Instituições. Porto Alegre: UFRGS.

Scholze, S. (2000). Regulamentação da proteção e transferência de tecnologia. In: Encontro de Propriedade Intelectual e Comercialização de Tecnologia, 3. Anais...Rio de Janeiro: Rede de Tecnologia do Rio de Janeiro.

Schartzman, S. et al. (1995). Ciência e tecnlogia no Brasil: uma nova política para o mundo global. In: Schwartzman, S. Ciência e tecnologia no Brasil: política industrial, mercado de trabalho e instituições de apoio. Rio de Janeiro: Fundação Getúlio Vargas, vol. 2.

Sherwood, R. M. (1992). Propriedade intelectual e desenvolvimento econômico. Tradução Heloísa Arruda de Vilela. São Paulo: Universidade de São Paulo.

Sierra Bravo, R. (2003). Tesis doctorales y trabajos de investigación científica; metodología general de su elaboración y documentación. Madrid: Thomson.

Stal, E. ; Fujino, A. (2005). Aprimorando as relações universidade-empresa-governo no Brasil; a lei de inovação e a gestão da propriedade intelectual. In: Seminário Latino Iberoamericano de Gestión Tecnológica, 11. Anais...Salvador: Altec. 


\section{Biblios La propiedad intelectual en las universidades brasileñas}

Theotonio, S. B. (2004). Proposta de implementação de um núcleo de propriedade intelectual e transferência de tecnologia no CEFET/RJ. 156f. Dissertação (Mestrado), CEFET/RJ, Rio de Janeiro.

Torkomian, A. L. (1997). Gestão de tecnologia na pesquisa acadêmica: o caso de São Carlos. 304f. Tese (Doutorado em Administração), Faculdade de Economia e Administração e Contabilidade, Universidade de São Paulo.

Vedovello, C. (1997). Science parks and university-industry interaction: geographical proximity between the agents as a driving force. Technovation, 17 (9), 491-502.

Viotti, E. B. (2003). Indicadores de ciência e tecnologia e inovação no Brasil. In: Viotti, E.B.; Macedo, M. M. (Org.). Fundamentos e evolução dos indicadores de C\&T. Campinas: Unicamp. 


\section{Biblios Intellectual property brazilian universities}

\section{Datos de las autoras}

Geni Anastacio Sabino

Graduada en Biblioteconomía por la Universidad Federal de Goiás (1991). Doctora por la Facultad de Comunicación y Documentación. Departamento de Información y Documentación Universidad de Murcia-España. Tiene experiencia en el área de Biblioteconomía con énfasis en Gestión de Bibliotecas y en el área de Ciencia de la Información, con énfasis en Procesos de Diseminación de la Información.

genisabino@hotmail.com

\section{Maria Dolores Ayuso García}

Profesora Titular de la Universidad de Murcia, pertenece al grupo de investigación sobre Políticas y Técnicas Aplicadas a la Sociedad del Conocimiento (SOCTEP). Además participa de la Cátedra María Moliner del Instituto Universitario "Agustín Millares" de Documentación y Gestión de la Información Universidad Carlos III, Madrid, España.

mayu@um.es

Recibido-Received : 2012-06-20

Aceptado-Accepted : 2012-09-29

\section{(c) $)$ BY-NC-ND}

This work is licensed under a Creative Commons

Attribution-Noncommercial-No Derivative Works 3.0 United States License.

\section{ULIS D-Sunte}

This journal is published by the University Library System of the University of Pittsburgh as part of its $\underline{D-S c r i b e}$ Digital Publishing Program and is cosponsored by the University of Pittsburgh Press. 